\title{
¿De qué hablamos cuando nos referimos al paramilitarismo?
}

Recibido: abril de 2017 Revisado: mayo de $2017 \quad$ Aceptado:junio de 2017

1 Docente investigador de la Universidad de San Buenaventura, Medellín. Director del grupo de investigación GIDPAD. Contacto:

Alfonso.insuasty@usbmed. $\underline{\text { edu.co }}$

ORCID

http://orcid.org/0000$\underline{0003-2880-1371}$
Por: Alfonso Insuasty Rodriguez ${ }^{1}$

\begin{abstract}
Resumen
Se trata de una estrategia cuyo centro es la aplicación de fuerza a escala, en la búsqueda de homogenizar modelos sociales, políticos, económicos, valóricos, hasta espirituales. Es así que, a esta aplicación de violencia o pedagogía reordenadora social basada en el terror, le antecede y le sigue, una planeación del desarrollo, un riguroso análisis prospectivo de la economía "global". Todo se calcula, se proyecta, se mide, de tal forma que toda acción emprendida para el fin de homogenizar sociedad entera, se vaya conectando en términos de tiempos, dinámicas y resultados.
\end{abstract}

Palabras clave. Reordenar la sociedad; captura del territorio; paramilitarismo; cultura paramilitar

\section{Contexto}

El despojo y la expropiación han sido elementos fundamentales para el reordenamiento económico, político y sociales y la imposición de un nuevo modelo. En Europa por ejemplo, esto se dio a través de la acumulación por despojo, que se basó en la expropiación violenta a los productores de sus condiciones de producción Vega Cantor \& Martín Novoa (2016) "se presentó en Inglaterra en el momento de formación del capitalismo no fue exclusiva de su época y país, sino que se sigue presentando hoy como un mecanismo de funcionamiento del capitalismo en su expresión mundial". 
En Colombia, según ambos autores, el despojo, la violencia y la expropiación se ha ensañado contra los indígenas, campesinos y afrodescendientes desde hace más de un cuarto de siglo.

Lo cual ha significado que les se han arrebatados a esos sectores sus tierras, (unos seis millones hectáreas), sus ríos y sus bosques, que han pasado a manos de empresarios capitalistas, narcoparamilitares y multinacionales. En ese proceso de expropiación, las cuales dominantes han recurrido a todos los procedimientos violentos de despojo, lo cual debería asombrar y producir vergüenza al mundo. Como resultado Colombia ocupa el primer lugar mundial en cuanto al número de despojados internos, con una cifra de cinco y medio millones de personas, la mayor parte de ellos campesinos e indígenas. Este proceso de despojo violento. (Vega Cantor \& Martín Novoa, 2016)

Se trata de todo un ejercicio de poder y fuerza que buscaba y busca "reordenar la sociedad y sus territorios". ¿Cómo comprender ese "Reordenar la sociedad y sus territorios? por un lado, se trata de hacerse a aquellos territorios estratégicos para la producción, por otro, de reordenar la mente, el sentir, el pensar de comunidades distantes a dichos intereses, a dichas cosmovisiones. Pasa esta estrategia por la construcción de relatos justificadores, hasta acciones contundentes de fuerza y de procesos "educativos", mediáticos, culturales, espirituales, de consolidación de su objetivo en el tiempo.

¿Quién reordena? El poder hegemónico dominante, que suele ser una alianza entre Estado, Sectores Empresariales, Militares, Elites locales y Mafia.

Es toda una estrategia bien estructurada, más allá de la acción militar y paramilitar - siendo su centro -, es una sumatoria de acciones aparentemente desarticuladas, pero bien coordinadas, de aplicación constante, permanente, con gran capacidad de adaptación a las realidades cambiantes de su entorno.

Busca esta estrategia, con el reordenamiento del territorio:

- $\quad$ La activación de un tipo de economía que se ha dado en llamar "global” que no es más que la economía anglosajona y europea como modelo.

- Reordenar el pensar de la sociedad, su sentir, su visión de mundo, su pensamiento, intereses, sueños, deseos, formas de habitar una región geográfica determinada, maneras de significar, de simbolizar, de definir incluso el uso de sus suelos, sus costumbres, economía, maneras de consumir, redefine el rol de sus habitantes, de hombres, mujeres, niños, niñas, etc.

- Y lo más preocupantes es que siempre busca reordenar en un tiempo corto, no prolongado, pues el tiempo en el mercado "global" es dinero, lo que supone, la aplicación de toda una pedagogía del terror, a la que le sigue la consolidación o la captura incluso, de lo que llamamos el "sentido común" de un pueblo

- $\quad$ Es una estrategia capaz de analizar y calcular su entorno, sus lógicas para adecuarse y mutar en su lenguaje, su semántica, pero con el mismo significado, actúa a modo de hidra.

Ésta Estrategia que se desarrolla gracias a una estructurada pedagogía social, está compuesta por varios elementos, entre ellos:

- $\quad$ Pedagogía del terror, es la estrategia central, primera, de aplicación directa y luego en dosis pequeñas y permanente, violación constate de los derechos humanos, de la dignidad de los pueblos, destrucción del cuerpo, de la historia, del recuerdo, instaurar un relato de vencedores y vencidos, etc., así, se reordena la distribución poblacional en los territorios y todo 
en función del nuevo y útil uso de estas zonas geográficas, según el dictamen de ese "mercado global".

- Pedagogía del no-sentido de la estupidez, para la construcción de individuos competitivos, que sueñan en ser exitosos, alcanzar cosas, tener, ascender socialmente, separarse de sus pares sociales.

- Pedagogía de la desesperanza, nada es posible hacer, mejor será adaptarme lo mejor posible y sacarle lo más que pueda a la situación. Individuos que piensan que ya todo está dado, que es necesario cambiar nada, que es inútil intentarlo.

Su construcción es visible, palpable, se ha gestado durante décadas, logrando:

- Una permanente construcción de la inferioridad, es inferior toda cultura, toda persona, todo grupo, toda forma organizativa que no se alinee a este modelo del "mercado global", toda forma que no se parezca a la anglosajona y europea como modelo, e incluso pervive la construcción del inferioridad de toda lengua, cultura, formas de relacionamiento cultura, deporte, etc., que no se parezca al "modelo", todo sueño y deseo que no se acerque al estándar establecido., del "gran garrote" de una educación dada para comprender que eso que se hace de alguna forma "está bien”, se acompaña de una narrativa particular, que hace ver como aceptables los resultados de dicha aplicación de fuerza para el reordenamiento de esas colectividades humanas para fines de un modelo económico específico. Esta inferioridad se consolida en la escuela, en las iglesias, en los masivos medios de comunicación, en los estándares de "seres humanos" exitosos, el modelo de ser hombre o mujer, niño o niña, todo significa y simboliza quien entra en la categoría inferior o quien logra saborear un aire de superioridad.

- Una sociedad colmada de individuos competitivos. "sálvese quien pueda" será el relato ordenador ya no externo, sino muy interno, hecho piel, hecho "sentido común social", naturalizado incluso eso que llamamos "caridad" como la máxima expresión de compasión ante los pobres que serán los otros, aunque yo sea pobre. Se desarticula así el sujeto, para ser individuo, uno aislado, desconectado de los otros, de su historia, sin la información ni formación suficiente para hacerse preguntas de sentido sobre su entorno, sin capacidad para digerir lo que le ocurre y ocurre en su entorno.

- $\quad$ El fin último es ascender socialmente. Un sujeto desconectado de si, de su historia, de sus valores colectivos, requiere de un relato ordenador que le dé sentido, así aparece una suerte de "coaching social", un pseudo relato religioso que hiperactiva el proyecto de individuos que miden su crecimiento en el tener, en el éxito socio-económico, verdaderos ejércitos defensores a ultranza del sistema, asumiendo un lenguaje que en la mayoría de casos es irreflexivo, defendiendo lo ilógico, lo no-ético, así en su discurso externo manifiesten querer otro mundo posible.

Se trata de una estrategia cuyo centro es la aplicación de fuerza a escala, y que se surte además de varias sub-estrategias que en el tiempo van dando resultados en la búsqueda de homogenizar modelos sociales, políticos, económicos, valóricos, hasta espirituales.

Es así que, a esta aplicación de violencia o pedagogía reordenadora social basada en el terror, le antecede y le sigue, una planeación del desarrollo, un riguroso análisis prospectivo de la economía 
"global". Aquí todo se calcula, se proyecta, se mide, de tal forma que toda acción emprendida para el fin de homogenizar sociedad entera se vaya conectando en términos de tiempos, dinámicas y resultados.

Este ejercicio homogeneizador y re ordenador de las comunidades y sus territorios, inoculó entre otras, un sentir común de la realidad como lo dado e inmodificable en un grueso número de habitantes de poblaciones como América Latina, África, Asia, habitantes del Sur Global que han sufrido y sufren hoy la aplicación de violencia o pedagogía reordenadora social basada en el terror.

Para tratar de construir elementos de análisis sobre estas dinámicas de reordenamiento social, territorial, nos remitiremos Mediados del siglo XX, resaltando algunas ideas punteadas para el análisis y la discusión.

\section{¿Qué nos deja la II Guerra Mundial?}

- Diversas formas y técnicas de aniquilamiento de la población, de ejércitos convencionales a ejércitos mercenarios.

- Se transita de guerra entre Estados a otras formas de confrontación interna, entre poblaciones o grupos, y ya no contra el Estado sino autoridades del Estado o poderes regionales que capturan las funciones del Estado. - Romero -

- Se transita hacia una guerra irregular, una confrontación que se conoció como Guerra Fría, potencias enfrentadas a través del apoyo a fuerzas irregulares en cada región de interés. Se viven reales guerras de exterminio como "guerras ajenas"

- Se instaura un tipo de economía que golpea el sur como abastecedor del norte, lo que genera reordenamiento de los territorios, en tiempos cortos, en función de un modelo económico foráneo, ganado gracias a posiciones de fuerza y captura de las decisiones de dichos estados gracias a fuertes alianzas con las élites locales.

- Transitamos de conflictos entre Estados a conflictos internos, de conflictos netamente rurales, a urbanos, y hacia formas hibridas de administración de la violencia:

- Se vive un desvanecimiento de las fronteras que separan a combatientes y civiles,

- La aplicación de técnicas de contrainsurgencia para sembrar el odio y terror en la población,

- El control social,

- La violación sistemática de los derechos humanos, hasta su cuasi naturalización

- La modificación de los campos de batalla

- Modos de financiación de los diversos conflictos devenidos de las diversas formas de financiación de las Guerras, que pueden ser una mezcla de economías ilegales, métodos extorsivos o financiación de señores de la guerra, mafias o empresarios de la coerción (Romero, 2003).

- Ruptura del tejido social y el control de la población por medio de la administración del miedo y del terror.

\section{Aplicación de una dura Doctrina Militar: Doctrina de Seguridad Nacional.}


El mundo, con estos aprendizajes y una bipolaridad global, se sume en una estrategia global de control, del miedo, de la sospecha, de la idea del "enemigo".

Este control, se acompaña de algunas estrategias:

\section{Estrategia Contrainsurgente}

La Doctrina de Seguridad Nacional (DSN), se aplicó como doctrina contrainsurgente en Latinoamérica, específicamente en los países del Cono Sur durante las dictaduras militares.

Esta Doctrina contrainsurgente fue creada por los Estados Unidos a inicios de los años sesenta con el fin de:

- Ejecutar la llamada "guerra de contrainsurgencia".

- Un modelo para enfrentar los conflictos sociales, defendiendo el orden social tradicional o imponiendo un nuevo orden afín a los intereses trazados por el modelo económico capitalistas.

\section{"Enemigo interno"}

El enemigo en sí son las ideas y practicas ajenas al modelo económico capitalista.

Encarnan esa pugna por otro sistema en su momento, la izquierda armada, pero se extiende esta categoría de enemigo interno a todo aquel que propugnara por un orden social contrario a los intereses en esencia económicos (políticos, sociales, culturales).

\section{"Guerra total"}

Vencer al enemigo interno a cualquier precio, por medio del uso de estrategias diversas de orden económicos, diplomáticos, psicológicos, mercenarios, mediáticos y culturales.

Esto implicó, para el gobierno de los Estados Unidos, el aumento de ayuda económica vía empréstitos y apoyo a ONG`s (USAID) y militar a gobiernos y/o grupos contrainsurgentes paramilitares, mercenarios, narcotraficantes- alineados a favor de esta potencia y su fin mercantil.

Para hacerle frente a este tipo de guerra, el orden doctrinario se recurrió a la aplicación de acciones contrainsurgentes como:

- Armar a la población en grupos de autodefensa,

- Ofrecer entrenamiento militar a grupos contrasubversivos,

- Promover campañas de desinformación y crear redes de informantes (Kornbluh, 1988;

Klare, 1988). 


\section{La población civil en este tipo de guerra es el blanco de la disputa.}

Propagar las ideas subversivas en la población eran aún más peligrosas que las mismas armas. En lo operativo, la DSN privilegió los aspectos psicosociales destinados a cambiar en la población, por medio de la represión, las ideas consideradas negativas de cambio social.

De esta forma, la DSN se convirtió, en la mayoría de los países, en la herramienta de las clases dominantes (locales y extranjeras) para obtener fines económicos para lo cual fue funcional la división de la sociedad en amigos y enemigos (Cfr. Izaguirre, 2009).

\section{Propaganda}

Se buscaba restringir la información que se difundía los medios de comunicación, la contratación de "cazadores profesionales" para perseguir y eliminar a los "terroristas"-mercenarios-, difundir información falsa que desacredite al enemigo (guerra psicológica) y la validación de actividades paramilitares encubiertas.

Persuasión ideológica y psicológica, la conformación de amplias redes de espionaje y la subversión como marca de identificación que justifica la eliminación del enemigo.

En el campo de la acción militar se consideró necesaria la ampliación de las tareas de inteligencia, el entrenamiento de las fuerzas militares tercermundistas en la guerra de guerrillas y el apoyo de las fuerzas militares en grupos paramilitares, a los cuales debían armar y entrenar.

Se redefine las funciones de las fuerzas militares - defensores y encargados de recuperar el orden limpiando a la sociedad del enemigo interno.

\section{Estructura legal estatal}

Un Estado con normas e instituciones, manejos, adaptas a estos fines. Se asume a ello que el Estado tiene la función de guiar a la sociedad, de proporcionarle las directrices para su desarrollo y de protegerla de los peligros [...] Para poder cumplir cabalmente con la función prioritaria de seguridad, el gobierno debe acumular en sus manos todos los recursos existentes: políticos, económicos, militares, sociales y psicológicos (Leal, 2002, 17).

La función de guía recae entonces en las fuerzas militares, las cuales, al considerarse las defensoras de la nación, creyeron pertinente incluso, "controlar el gobierno" cuando percibieron que la seguridad nacional se hallare amenazada. Lograron así la imposición del terrorismo como práctica política que consiguió "no solo identificar y destruir al enemigo actual [los grupos de guerrillas], y disuadir a los enemigos potenciales, sino convencer al ciudadano común de que su seguridad personal es función inevitable y obligada de su incondicionalidad frente al régimen” (Leal, 2002, p.18).

La aplicación de la DSN es afín al nuevo orden económico-social capitalista por ende busca acabar ahora y siempre todo "germen de insurgencia ideológica anticapitalista" Se buscan múltiples estrategias para imponer un orden social que sustituyera todo lo que pudiera asemejarse al modelo económico socialista. 


\section{¿Por qué estas ideas calaron en América Latina y en Colombia?}

Gracias a la unificación de intereses defensivos de élites criollas y multinacionales estadounidenses y europeas, contra las demandas sociales de los trabajadores fuertemente influenciados por la izquierda radical. Lo que, de acuerdo con la lógica de radicalismo ideológico de la Guerra Fría, suscitó en los grupos de propietarios temor y sentimientos de promover la defensa radical de sus intereses, consolidándose así un sistema de alianzas entre militares, paramilitares-mercenarios, apoyados por la Central Intelligence Agency (CIA), grupos dominantes y multinacionales. Relación que aún hoy continua.

\section{Colombia: el anticomunismo radical y la concepción del enemigo interno}

Colombia ha tenido una configuración cultural proclive a este tipo de guerras, ideas, estrategias, de hecho, así sucedió durante la guerra bipartidista, las élites que encarnaban estos partidos que al mismo tiempo eran los grandes tenedores de tierras y comerciantes, ejes de la economía del país, utilizaron en Estado como forma de dominación, exterminio del opositor y de implantar sus ideas y proyectos, creando fuerzas legales militares y fuerzas ilegales armadas para tales fines.

Ya, luego de la segunda Guerra, entramos directamente a cumplir funciones de "perros bravos" del sistema, de Estados Unidos y Europa, para evitar que el fantasma de las "ideas" contarías al sistema económico capitalista entraran al continente. A lo anterior, se suma una reacción constante de la Elite Regional y cúpulas militares ante treguas de paz ejemplo la firmada en 1983 entre el gobierno y algunos grupos de guerrillas.

Los intereses militares de lucha contrainsurgente coincidieron o favorecieron los intereses de distintos sectores sociales como los capos del narcotráfico (que ya venían invirtiendo recursos en seguridad privada para defenderse de las guerrillas, proteger sus territorios ganados de manera ilegal y para mantener sus inversiones en diversos sectores empresariales) y élites regionales que apoyaron con financiación, directrices, etc., la creación de grupos de civiles armados para hacerle frente a la lucha contrasubversiva

La iniciativa del Ejército benefició, sin duda, a los narcotraficantes, pues a la vez que enfrentaban a las guerrillas, que también les habían exigido pago de impuestos por cada cargamento de droga, la unión con los militares les permitía disponer de territorios seguros para su negocio sin tener que preocuparse por enfrentar a la fuerza pública.

A este sistema de muerte, económica y políticamente se les ofrecía ventajas como: legalización de los dineros, valorización de los territorios comprados especialmente en ganadería extensiva cambiando con ello el uso de los suelos e inserción en la escala social de las élites dominantes regionales.

Sobre las operaciones conjuntas entre las fuerzas militares y grupos paramilitares, en el año 2009 fue noticia nacional la desclasificación de documentos secretos divulgados por un grupo de investigadores de la Universidad de George Washington, en su sitio web llamado National Security Archive, en los que se comprueba que la CIA tenía conocimiento de que las Fuerzas Armadas colombianas cooperaban con grupos paramilitares. De acuerdo con estos documentos, en un informe 
de inteligencia de la CIA emitido en 1994, se determinó que las fuerzas de seguridad colombianas utilizaban "tácticas de escuadrones de la muerte en su campaña contrainsurgente". Los oficiales tenían, además, según ese informe, un historial de "asesinato de civiles de izquierda en áreas de control guerrillero, colaboración con paramilitares vinculados con el narcotráfico en ataques contra presuntos simpatizantes de la guerrilla, y el asesinato de combatientes capturados" (El Espectador, 2009, para. 6).

Visto así, desde una dimensión política, el paramilitarismo puede ser leído como reacción armada de una alianza política, económica y militar de grupos de poder local en defensa del statu quo; con un marcado interés en evitar, por medio de la violencia armada, la redefinición del sistema político y de reformas sociales como la distribución de la tierra. Visión que se complementa con la propuesta por Vilma Franco (2009) de caracterizar a los grupos paramilitares como una fuerza irregular a la que se le encomendó la "guerra contrainsurgente y mantenimiento o reconfiguración del orden interior"

\section{Una brevísima y general línea de tiempo}


Élites enfrentadas crean fuerzas legales (policía, ejercito, guardias de rentas, popol), e ilegales (pájaros, chulavitas, chusmeros y bandoleros)

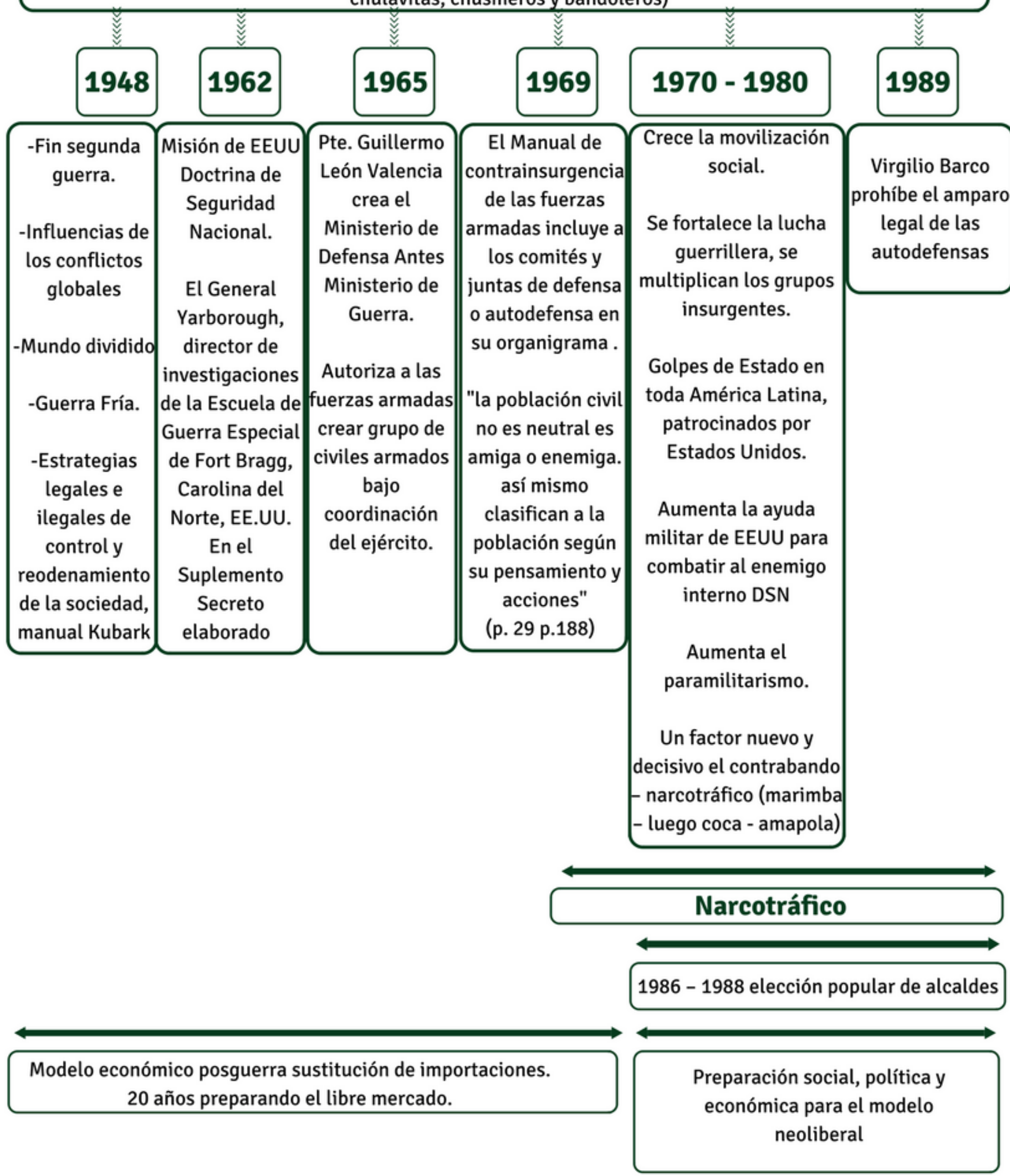

Fuente: Elaboración del autor 

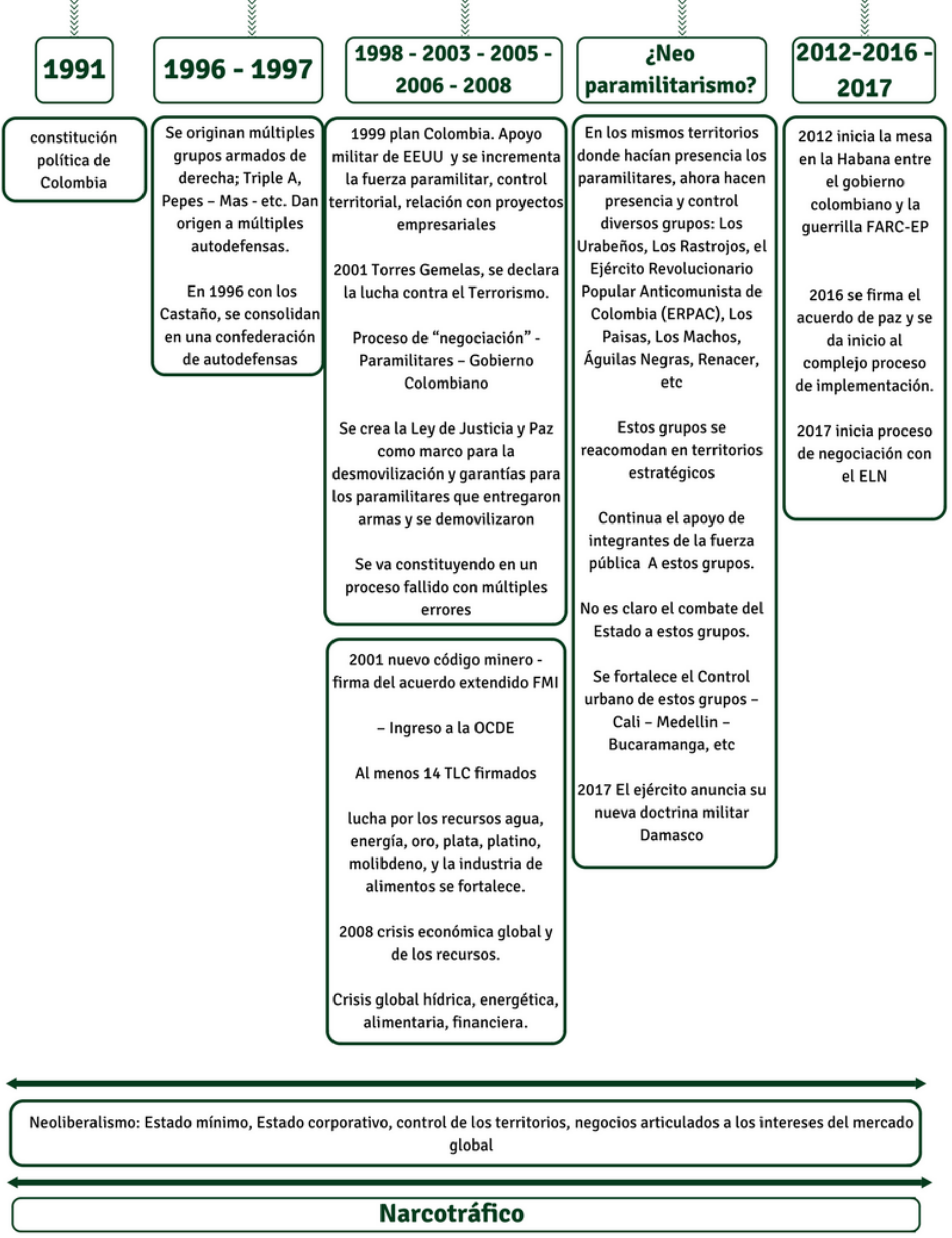

Fuente: Elaboración del autor 


\section{El paramilitarismo colombiano puede diferenciarse por}

La autonomía militar que lograron los grupos paramilitares gracias a su vinculación con el mercado de las drogas, como también al apoyo social, político y económico que recibieron de diversos sectores regionales.

Aun la fuerte alianza entre el paramilitarismo y la fuerza pública, su accionar fue más allá de las tareas de contrainsurgencia que estaban fuera del alcance del Ejército por limitaciones de tipo legal relacionadas con la legislación nacional e internacional de violaciones a los derechos humanos y al Derecho Internacional Humanitario.

Se convirtieron en una estrategia armada para la acumulación de riquezas personales por medio del despojo de tierras y su apoyo a grupos políticos y económicos para la acumulación a través del despojo y desplazamiento forzado, así como para triunfos electorales.

El monopolio de la economía ilegal drogas, minería, contrabando ha sido el botín de estos grupos para lo cual requieren mantener fuertes alianzas regionales políticas, sociales.

Colombia es un país con una "regionalización acentuada, dispersa y con tendencia endógena que dificulta la integración social, económica, política y de infraestructura para la formación de la unidad nacional" (Leal, 2012, para. 2).

Reacción política y armada funcional a los intereses de una alianza que integró a diversos sectores del poder regional, grupos económicos, comerciantes y sectores de la cúpula militar. Un marco de legalización estatal que favoreció la represión como forma de resolver los conflictos sociales y políticos, y el apoyo de las Fuerzas Armadas en ejércitos paraestatales como estrategia para combatir al enemigo interno.

\section{Hoy: hibridación del paramilitarismo como estrategia que pervive}

Se transita de una guerra rural de alta intensidad hacia una guerra sostenida urbano-rural para el control económico local ilegal alineado al control político-económico de cada barrio, comuna, vereda, corregimiento, etc.

Se ha hibridado la actividad económica ilegal con la legal por medio de inversiones en establecimientos de comercio locales como panaderías, juegos de azar "maquinitas", venta y distribución de huevos, arepas, etc.

Se ejerce un efectivo control territorial, efectivo pero diluido con vínculos obligados y hasta simbólicos, con las juntas de acción comunal, corporaciones, ONG`s, etc., una pugna por la captura incluso de los contratos con el Estado. "A mandado", se controla la participación política local

Este control permite alianzas con la autoridad local política y militar local

La circulación, legalización, lavado del dinero es un tema clave a tener presente en esta nueva etapa, ¿Cómo pueden circular y legalizar el dinero fruto de estas actividades? 
Durante la devastación de la guerra contrainsurgente en su reciente etapa en paralelo se consolidó una serie de reformas al Estado que lo hicieron el más fiel defensor de la inversión trasnacional, reformas paulatinas a la Educación moldeándola a dicho modelo económico empresarial "global" y unas reformas legales que dejaron como resultado la concentración de la información en dos grandes centros de poder mediático que controlan lo que se dice, lo que se piensa, lo que se opina en el país, lo que se ve en Tv y lo que se escucha en radio todo un sistema de control y dirección social que capturan el sentir, el pensar, el desear, el soñar, el opinar de toda una masa social. Esto es también, una clara estrategia de Control.

- Se crea una sensación de naturalización y desesperanza aprendida, nada es posible hacer, nada se puede cambiar, lo mejor es una lucha individual por alcanzar algo, lo mínimo para cada quien.

- Crece una nueva generación que buscan desconectar de sus historias y por ende de sus luchas como pueblo.

- Sigue una etapa de captura de los territorios, de amplias zonas dedicadas de lleno o a la agroindustria o a la actividad minero-energética dada a las grandes corporaciones transnacionales.

- Se acentúa, normaliza y naturaliza el accionar de estos grupos en zonas estratégicas mineras, de agroindustria, donde se traza y construcción mega-obras de infraestructura, apoyan incluso en tránsito de su vocación productiva, todo va favoreciendo el mercado a las lógicas globales de "reordenamiento social y productivo de los territorios".

Preocupa es la consolidación de una lógica cultural proclive a los intereses del mercado, que normalizar dichos valores del tener, el acumular, el despilfarro, la cultura narco-paramilitar, la consolidación de unas políticas que desindustrializaron de la región y del país generando mayor abandono social, crisis humanitaria, todo un caldo de cultivo para generar la inestabilidad que requiere de "otro orden", en los barrios y comunas. A la par, el sistema educativo básico y superior ya no forma sujetos capaces de comprender lo que ocurre, de pensar en su historia, de pensar en otras formas de construir sociedad, solo adapta al individuo al sistema, evitando masa crítica capaz de poner condiciones.

Hoy, a pesar de estos escenarios, se acentúa la esperanza, se articulan otras formas de hacer, de construir pensamiento crítico, se articulan diversos ejercicios por rescatar al sujeto, por reconfigurar tejido social desde el sentir propio de las comunidades, la defensa de lo público, por articular la investigación y la acción, la formación desde otros lugares a lo instituido, por la defensa de los bienes comunes, del agua, del medio ambiente, de la cultura, de la vida, las redes y articulaciones cobran mayor sentido, fuerza y vigor levantando su voz poco a poco, desde otros lugares a los "dados" y esto viene ocurriendo en múltiples regiones por toda América Latina.

\section{Referencias Bibliográficas}

ACA, A. C. (2003). Desplazados ¿Refugiados internos o excluidos? Medellín: ACA. Accion Contra Minas. (2016). Definición Internacional victimas MAP MUSE. Obtenido de accioncontraminas.gov.co: 
http:/ / www.accioncontraminas.gov.co/estadisticas/Paginas/victimas-minasantipersonal.aspx

Accion contra minas. (31 de julio de 2017). Victimas de Minas Antipersonal. Obtenido de Accion contra minas: http://www.accioncontraminas.gov.co/estadisticas/Paginas/victimas-minasantipersonal.aspx

Bastidas, W., Carrion, J., Balbin Alvarez, J. W., \& Insuasty Rodriguez, A. (2010). Las Victimas en Contextos de Violencia e Impunidad: Caso Medellín. Medellìn: Instituto Popular de Capacitaciòn.

Behar, O. (2011). El clan de Los Doce Apóstoles. Bogotá: Ícono.

Cadavid , P., Insuasty Rodriguez , A., Restrepo , L., \& Balbin , J. (2009). Victimas, violencia y despojo. medellin: IPC. Obtenido de http://web.usbmed.edu.co/usbmed/formacion/docs/victimas.pdf

CCEEU. (2016). Entre el sueño de la Pazy la continuidad de la Guerra. Informe Derechos Humanos 2016. Medellin: CCEEU.

CCOA. (20 de febrero de 2016). Generamos un ambiente propicios para los negocios. Obtenido de Camara de comercio del Oriente Antioqueño: https://www.ccoa.org.co/contenidos/categorias/oriente_antioquenio.php

CJL, C. J. (2012). Caminando en La Esperanza por Justicia y dignidad. Medellín: Nuevo Milenio.

CNMH, C. N. (2010). Silenciar la democracia. Las masacres de Remedios y Segovia 1982-1997. Bogotá: Taurus.

Corporación Jurídica Libertad. (20 de noviembre de 2012). Comisión en la Esperanza por justicia y dignidad. Medellin: Corporación Jurídica Libertad. Obtenido de Coporacion Jurídica Libertad: http://www.cjlibertad.org/files/Caminando\%20en\%20la\%20Esperanza.pdf

Gómez, J. D. (2013). Consecuencias psicosociales de la participación en escenarios de justicia transicional en un contexto de conflicto, impunidad y no-transición. El Ágora USB, 307-338.

Grisales González, D., \& Insuasty Rodriguez, A. (2016). Mineria, conflictos y derechos de las victimas. Colombia. Medellín: Editorial Kavilando.

Grisales González, D., Insuasty Rodriguez, A., \& Gutierrez Leon, E. (2013). Conflictos asociados a la gran mineria en Antioquia. El Agora USB, 13(1), 371-397. Obtenido de http:// revistas.usb.edu.co/index.php/Agora/article/view/213/94

ILSA, I. L., FCSPP, F., \& MOVICE, M. (2009). Desplazamiento Forzao y retorno en San Carlos Antioquia. Una comunidad que regresa hacia el confinamiento. Bogotá: Gente Nueva.

Incoder. (s.f.). Estrategia de desarrollo rural. Obtenido de Incoder: http://www.incoder.gov.co/documentos/Estrategia $\% 20 \mathrm{de} \% 20$ Desarrollo\%20Rural/Pertile $\mathrm{s} \% 20$ Territoriales/ADR_URABA/Documentos $\% 20 \mathrm{de} \% 20$ apoyo/DNPAtrato $\% 20 \mathrm{y} \% 20 \mathrm{Ur}$ aba/3c23PlanUraba4.pdf

Insuasty Rodriguez, A., Henao Fierro, H., Pino Franco, Y., Borja Bedoya, E., Barrera Machado, D., Sanchez Calle, D., \& Mejìa Murillo, M. (2016). Murindò. Medellìn: Kavilando.

Insuaty Rodriguez, A. (16 de marzo de 2017). "Nadie nos defendía". Exterminio de la Unión Patriótica. Colombia. Obtenido de kavilando: http://kavilando.org/index.php/2013-10-13-19-5210/conflicto-social-y-paz/5372-nadie-nos-defendia-exterminio-de-la-union-patrioticacolombia 
IPC, I. P. (2015). Territorialidad, poder, conflicto y par: Medellín: IPC.

Legiscomex.com. (14 de Agosto de 2013). ¿Aumentará la competitividad de Medellín con la construcción del

Puerto de Urabá? Obtenido de legiscomex.com:

http://www.legiscomex.com/BancoMedios/Documentos\%20PDF/distribuci $\% \mathrm{C} 3 \% \mathrm{~B} 3 \mathrm{n} \% 2$ 0f\%C3\%ADsica $\% 20$ internacional-puerto $\% 20 \mathrm{de} \% 20$ urab $\%$ C3\%A1.pdf

Molano, A. (24 de Enero de 2009). La socia mandé norte. Obtenido de El Espectador:

http://www.elespectador.com/choco/columna111273-socia-mande-norte

Mujica Chirinos , N., \& Rincón González , S. (2006). Concepciones del desarrollo en el Siglo XX:

Estado y Política Social. Revista de Ciencias Sociales (Ve), XII(2), 205-222. Obtenido de

http:/ / redalyc.uaemex.mx/src/inicio/ArtPdfRed.jsp?iCve=28011651002

Observatorio de Paz y Reconciliación del Oriente Antioqueño. (2006). Boletin \#1. Rionegro: Observatorio de Paz.

Observatorio de Paz y Reconciliación del Oriente Antioqueño. (2007). Boletín \#2. Rionegro: Observatorio de Paz.

Observatorio de Paz y Reconciliación del Oriente Antioqueño. (2008). Boletin \# 4 informe 2007. Rionegro: Observatorio de Paz y Reconciliación del Oriente Antioqueño.

Observatorio de Paz y Reconciliación del Oriente Antioqueño. (2008). Boletín \# 6 Mapa Político en el Oriente Antioqueño 1988-2008. Rionegro: Observatorio de Paz y Reconciliación del Oriente Antioqueño.

Observatorio de Paz y Reconciliación del Oriente Antioqueño. (2008). Oriente: memoria desenterrada. Rionegro: Observatorio de Paz y Reconciliación del Oriente Antioqueño.

Ochoa, F., Smith Quintero, R., \& Villegas Botero, L. J. (2002). El sector ele $\square$ ctrico colombiano : or $\square$ genes, evolucio $\square$ ny retos o un siglo de desarrollo, 1882-1999. Bogota $\square$, D.C. : Interconexio $\square$ n Ele $\square$ ctrica S.A.

Olaya Rodríguez, C. (2012). Nunca mas contra nadie. Medellín: Cuervo Editores.

Olaya Rodriguez, C. H. (2016). Conflictos socio-ambientales en el Oriente Antioqueño. Kavilando, 8(2), 15-21. Obtenido de http://kavilando.org/revista/index.php/kavilando/article/view/28/17

Olaya Rodriguez, C. H. (2017). El exterminio del Movimiento Cívico del Oriente de Antioquia. El Agora USB, 128-144. doi:http://dx.doi.org/10.21500/16578031.2815

Olaya, C. (2012). Nunca más contra nadie. Medellín: Cuervo Editores.

Orrego, L. P. (2008). La memoria decapitada. Espacio y estética en los asentamientos de desplazados de la ciudad de Medellin. Medellín: IDEA.

OSH, O. d. (2014). Nuestras voces sobre seguridad bumana en Medellin. Medellín: Universidad de Antioquia.

Ruíz, C. (2000). Un Pueblo que lucha: el Oriente Antioqueño.

Serrano, A. (2010). La multinacional del Crimen. La tenebrosa oficina de Envigado. Bogotá: Debate.

Suárez, J. E. (2016). Colombia nunca más Crímenes de lesa humanidad en la comuna trece. Medellín: Léanlo.

Unidad Nacional de Atención y Reparacion a Victimas del conflicto Armado. (2016). Cirfras Unidad de Victimas. Obtenido de unidadvictimas.gov.co:

http://cifras.unidadvictimas.gov.co/Home/Vigencia 
Universidad de Antioquia \& Corporación Jurídica Libertad CJL. (2012). Crímenes de Lesa Humanidady de guerra. Medellín: Universidad de Antioquia.

Valencia Grajales, J. F., Insuasty Rodriguez, A. \&, Agudelo Arango, J. J., (2017). Elementos para una genealogía del paramilitarismo en Medellin. Medellin: Kavilando.

Valencias Grajales, J. F., Insuasty Rodriguez, A., \& Restrepo Ramirez, J. d. (2016). Elementos para una genealogía del paramilitarismo en Colombia. Medellin: Kavilando. Obtenido de http://biblioteca.clacso.edu.ar/Colombia/kavilando/20170210032121/0.pdf

Vega Cantor, R. (2012). Colombia en la geopolitica hoy. El Ágora USB, 367-402. doi:http://dx.doi.org/10.21500/16578031.81

Vega Cantor, R., \& Martín Novoa, F. (2016). Geopolítica del despojo. Bogotá: Cepa Editores.

Villa Gómez, J. D. (2013). Memoria histórica desde las Victimas del conflicto armado Construcción y reconstrucción del sujeto político. Kavilando, 5(1), 11-23. Obtenido de http://kavilando.org/revista/index.php/kavilando/article/view/96/82

Villa Gómez, J. D., \& Insuasty Rodriguez, A. (2016). Entre la participación y la resistencia: reconstrucción del tejido social desde abajo, más allá de la lógica de reparación estatal. El Agora USB, 453-478.

Villa Gómez, J. D., \& Insuasty Rodriguez, A. (2016). Significados en torno a la indemnización y la restitución en víctimas del conflicto armado en el municipio de San Carlos. El Ágora USB, 16(1), 165-191. Obtenido de http:/ / revistas.usb.edu.co/index.php/Agora/article/view/2171/1899

Viva la Ciudadanía. (20 de enero de 2007). La ruta de la expansión paramilitar y la transformacion de Antioquia. Obtenido de Viva la ciudadania: http://viva.org.co/cajavirtual/svc0080/articulo10.pdf 


\section{What do we talk about when we refer to paramilitarism?}

Received: april of $2017 \quad$ Reviewed: may of $2017 \quad$ Accepted: juny of 2017

By: Alfonso Insuasty Rodriguez ${ }^{1}$

1 Docente investigador de la Universidad de San Buenaventura, Medellín. Director del grupo de investigación GIDPAD. Contacto:

Alfonso.insuasty@usbmed. $\underline{\text { edu.co }}$

ORCID

http://orcid.org/00000003-2880-1371

\begin{abstract}
.
It is about a strategy whose core is the use of force to scale, in search of homogenizing social, political, economic values, and even spiritual models. So much so, that this use of violence or restructuring social pedagogy, which is based on terror, precedes it and follows it, a development planning, a rigorous prospective analysis of the "global" economy. Everything is calculated, projected, and measured, so that every action undertaken in order to homogenize society, as a whole, is linked in terms of times, dynamics, and results.
\end{abstract}

Key words: To restructure Society; Apprehension of the Territory; Paramilitarism; Culture

\section{Context}

Both dispossession and expropriation have been fundamental elements for the economic, political, and social restructuring, and the imposition of a new model. In Europe, for example, this was given through the accumulation by dispossession, which was based on the violent expropriation to the producers of their conditions of production, Vega Cantor \& Martín Novoa (2016): "It arose in England at the time of formation of capitalism and it was not exclusive of its time and country, but it is still present today as a mechanism of functioning of capitalism in its worldwide expression." 
In Colombia, according to both authors, dispossession, violence, and expropriation, have treated cruelty indigenous peoples, peasants, and Afro descent people from more than one quarter of a century ago.

This has meant that these sectors have been dispossessed of their lands (nearly six million hectares), lands, and forests, which have been passed down into the hands of capitalist tycoons, paramilitary drug traffickers, and multinationals. In that expropriation process, the dominant classes have resorted to expropriation violent procedures, which would be astonishing and embarrassing to the world. As a result, Colombia is at the forefront of the number of internally displaced populations, with a figure of 5.5 million people, most of which are peasants and indigenous peoples. This violent dispossession process. (Vega Cantor \& Martín Novoa, 2016)

It is about a real exercise of power and strength, which sought and seeks "to rearrange society and its territories." How can the "restructuring of society and its territories be interpreted?" On the one hand, it is about taking possession of those strategic territories for production purposes. On the other hand, it is about the restructuring of the mind, the deep feeling, and the thinking of distant communities to such interests, to such worldviews. This strategy goes through the construction of justified stories, and even, resolute actions of force and "educational," media, cultural, spiritual, and consolidation processes of its objective in time.

Who is the one in charge of reorganizing? The hegemonic dominant power, which is usually an alliance between the State, Entrepreneurial Sectors, the Military, Local Elites, and Mafia.

It is a really well-structured strategy, which beyond the military and paramilitary action -being its core,- is the sum total of apparently unarticulated, but well-coordinated actions, of constant and permanent application, with a great capacity of adaptation to the changing realities of its environment.

With the restructuring of the territory, this strategy aims at

- $\quad$ activating a type of economy, which has been called itself "global," but it is not just the Anglo Saxon and European economy as a model.

- $\quad$ restructuring a society's ways of thinking, feelings, worldviews, thoughts, interests, dreams, wishes; ways of inhabiting a given geographic region, ways of meaning, of symbolizing, of defining even the use of its soils, customs, economy, consumption patterns, redefines the role of its inhabitants: men, women, boys, and girls, etc.

- $\quad$ And what is more worrying is that it always seeks to restructure, within a short period since time in the "world" market represents money, what supposes the application of a theory of fear, which is followed by the consolidation or even the capture, of what is called the "common sense" of a people.

- $\quad$ It is a strategy, which is able to analyze and calculate its environment, logic for adaptation and to mutate into its language, its semantics. But with the same meaning, it acts as a hydra.

This strategy, which is developed thanks to a structured social pedagogy, is made up of several elements, among them, the following stand out: 
- Pedagogy of fear: It is the core and prime strategy, of direct application, and then, in small doses, and permanent and constant violation of human rights, of peoples' dignity, destruction of the body, of history, of memoirs, of the establishment of stories of winners and losers, etc. It is in this way that the population distribution in the territories is rearranged, and primarily on the basis of the new and useful purpose of these geographic zones, according to the report of that "world market."

- Pedagogy of Non-sense: That of stupidity, for the construction of competent individuals, whose dream is to become successful, to achieve things, to possess objects, to achieve upward mobility, to split up with their social peers.

- Pedagogy of hopelessness: Nothing can be done. It is better to adapt to circumstances, in the best possible way and to the greatest extent. It is necessary to change nothing. It is useless to give it a try.

Its construction is visible and touchable. It has been taking shape by decades, achieving:

- A permanent construction of inferiority: It is lower than every culture, every person, every group, every organizational form, which is not aligned with this "global market" model, any form that does not look like the Anglo-Saxon and European as a model, and even survives the construction of the inferiority of every language, culture, forms of relationship, sport, etc., which does not look like the "model," every dream and desire, which does not resemble the established standard, of the "big stick" of a given education to understand that what is done is somehow "okay." It is accompanied by a particular narrative, which makes the results of the application of force be acceptable to the restructuring of these human communities for the purposes of a specific economic model. This inferiority is consolidated in the school, in churches, in the mass media, in the standards of successful "human beings." The model to be a man or a woman, a boy or a girl, all this means and symbolizes who falls into the category of lower or who can enjoy an air of superiority.

- A society filled with competitive individuals. "every man for himself" will be the guiding story, no longer external, but very internal, who is made of skin, made of "social common sense," naturalized; even that which we call "charity" as the ultimate expression of compassion to the poor who will be the others, although I may be poor. The subject is then dismantled to become an individual, one who is isolated, disconnected from each other, from his history, without information or sufficient training to ask himself nonsense questions about his surroundings, unable to digest what happens and does not happen in his environment.

- The ultimate purpose is to achieve upward mobility. Thus, a subject, who does not have contact with himself, his history, his collective values, requires a guiding story, which gives him meaning, a sort of "social coaching," a pseudo-religious story appears that boosts the project of individuals, who measure their growth based on possessions, on the socio-economic success, on the real defending armies of the system, at any cost; adopting a language, which in 
most cases is thoughtless, defending the illogical, the unethical, even if in their external speech, they declare to want another possible world.

It is about a strategy whose core is the use of force to scale, in search of homogenizing social, political, economic values, and even spiritual models. So much so, that this use of violence or restructuring social pedagogy, which is based on terror, precedes it and follows it, a development planning, a rigorous prospective analysis of the "global" economy. Everything is calculated, projected, and measured, so that every action undertaken in order to homogenize society, as a whole, is linked in terms of times, dynamics, and results.

This homogenizing and reorganizing exercise of communities and their territories, injected, among others, a common sense of reality as given and unchangeable, in a large number of inhabitants of populations, such as Latin America, Africa, Asia, as well as inhabitants of the Southern Hemisphere, who have suffered and still suffer today the use of violence or restructuring social pedagogy, which is based on terror.

To try to build elements of analysis on these dynamics of social and territorial rearrangement, we will refer to the Mid-20th century, highlighting some ideas dotted for analysis and discussion.

\section{What did the Second World War leave behind?}

- Various forms and techniques of annihilation of the population, from conventional armies to mercenary armies.

- A war between States changes to other forms of internal confrontation, between populations or groups, not anymore against the State, but authorities of the State or regional powers, which take over the functions of the State. -Romero-

- An irregular war turned into a confrontation, which became known as the Cold War, embattled powers through the support to irregular forces in each region of interest. Real wars of extermination like "alien wars," are fought.

- A type of economy is established, it is one that hits the South as a provider of the North, which generates a rearrangement of territories, in short time, on the basis of a foreign economic model, gained from positions of strength and capture of the decisions of those States thanks to strong alliances with local elites.

- Conflicts between States change to internal conflicts, from purely rural conflicts to urban ones, and toward hybrid forms of management of violence:

- There is a fading border, which separates combatants from civilians,

- The application of counterinsurgency techniques to sow hatred and terror in the population,

- Social control,

- The systematic violation of human rights, to their quasi naturalization

- The modification of battlefields 
- Modes of funding of the different conflicts derived from the various forms of financing of wars, which can be a mixture of illegal economies, extortion methods or financing of warlords, mafia, or entrepreneurs of coercion (Romero, 2003).

- Rupture of the social fabric and the control of the population through the administration of fear and terror.

\section{Application of a tough Military Doctrine: The National Security Doctrine.}

The world, with this learning and a global bipolarity, is thrown into a global strategy of control, fear, suspicion, of the idea of the "enemy."

\section{This control is accompanied by some strategies: Counterinsurgency strategy}

The Doctrine of National Security (DSN) was applied as a counterinsurgency doctrine in Latin America, specifically in the countries of the Southern Cone of Latin America, during the military dictatorships. This counterinsurgency doctrine was created by the United States at the beginning of the 1960s with the aim of:

- applying the so-called "war of counterinsurgency."

- designing a model to address social conflicts, defending the traditional social order, or imposing a new order related to the interests laid down by the economic model capitalism.

\section{“The Internal Enemy"}

The enemy in and of itself are the ideas and practices alien to the capitalist economic model. They embody that struggle by another system at the time, the armed left, but this category of internal enemy is extended to anyone who advocates for a social order, which is at its core, contrary to economic (political, social, cultural) interests.

\section{"Total war"}

Defeating the internal enemy, at any price, through the use of diverse strategies of the economic, diplomatic, psychological, mercenary, media, and cultural order.

For the government of the United States, this meant the increase in financial aid by means of loans and support for NGO's (USAID) and military aid to governments and/or counterinsurgency groups - paramilitary people, mercenaries, and drug traffickers, who are aligned in favor of this power and its commercial purposes. 
To cope with this kind of war, the doctrinal order resorted to the application of counterinsurgency actions, such as:

Arming the population in self-defense groups,

Offering military training to counterinsurgency groups,

Promoting campaigns of disinformation and creating informant networks (Kornbluh, 1988; Klare, 1988).

\section{The civil population in this type of war is the target of the dispute}

Spreading subversive ideas within the population was even more dangerous than weapons themselves. In the operating level, the DSN favored psychosocial aspects for changing in the population, by means of repression, ideas of social change, of the negative order.

In this way, the DSN became, in most countries, the tool of the ruling classes (local and foreign) for achieving economic purposes, for which the division of society into friends and enemies was functional (cf. Izaguirre, 2009).

\section{Propaganda}

They wanted to restrict the information, which is disseminated by the media, the hiring of "professional hunters" to hunt down and to eliminate "terrorists" -mercenaries-, to spread fake information that discredits the enemy (psychological warfare) and the validation of covert paramilitary activities.

Ideological and psychological persuasion, the formation of extensive networks of espionage and subversion as brand identification, which justifies the elimination of the enemy.

In the field of military action, the extension of tasks of intelligence was deemed necessary, as well as the training of third-world military forces in guerrilla warfare and the support of the military in paramilitary groups, which were to be assembled and trained.

The functions of military forces were redefined - defenders and in charge of restoring order by cleansing society of the internal enemy.

\section{State legal structure}

A State with rules and institutions, management systems, adapt to these purposes. To this it is assumed that the State has the function of guiding society, of providing guidelines for its development and protecting it from the dangers [...] In order to fully meet the priority function of safety, the Government should accumulate in its hands all existing resources: political, economic, military, social and psychological (Leal, 2002, 17).

The guiding function then rests with the military forces, which considered to be the defenders of the nation, they believed relevant even, "controlling the government" when they realized that national 
security is found under threat. They managed so the imposition of terrorism as a political practice that managed "not only to identify and to destroy the current enemy [the guerrilla groups], and to deter potential enemies, but to convince ordinary citizens that their personal safety is an inevitable and forced function of its unconditional support against the regime" (Leal, 2002, p.18).

The application of the DSN is akin to the new economic and social order -the capitalist.- Therefore, it seeks to end now and forever all "anti-capitalist ideological insurgency germ." Multiple strategies are sought to impose a social order that would replace everything that might resemble the socialist economic model.

\section{Why did these ideas gather momentum in Latin America and in Colombia?}

Thanks to the unification of defensive interests of creole elites and American and European multinational against the social demands of workers strongly influenced by the radical left, which according to the logic of the Cold War ideological radicalism, caused in groups of owners fear and feelings of promoting radical defense of their interests, thus consolidating a system of military alliances, paramilitaries and mercenaries, supported by the Central Intelligence Agency (CIA), dominant and multinational groups. It is a relationship that still continues.

\section{Colombia: the radical anti-communism and the conception of the internal enemy.}

Colombia has had a cultural setup inclined to this kind of wars, ideas, strategies. In fact, this is what happened during the bipartisan war, elites who embodied these parties, and that at the same time, they were the major land holders and merchants, mainstay of the country's economy, used the State as a form of domination, destruction of the opponent, and the implementation of their ideas and projects, creating forces legal military and illegal armed forces for this purpose.

Well, after the Second World War, we went directly to perform functions of "fierce dogs" of the system, of the United States and Europe, to avoid that the ghost of the opposing "ideas" to the capitalist economic system came to the continent. Due to the above, in addition to a constant reaction of the Regional Elite and military leadership before peace truces. An example of this was the one signed in 1983 between the government and guerrilla groups.

Counter-insurgency military interests coincided or favored the interests of different social sectors, such as the drug lords (who were already investing resources in private security to defend themselves against guerrilla, protect their territories, which were gained illegally and to maintain their investments in various business sectors) and regional elites who supported with funding, guidelines, etc., the creation of groups of armed civilians to confront counter-insurgency operations.

The Army's initiative benefited, certainly, drug traffickers, because while they fought guerrilla, who had also requested payment of taxes by each drug shipment, the union with the military allowed them to make use of certain territories for their business without having to worry about confronting the security forces. 
To this system of death, economically and politically, advantages like these were offered: money laundering, valorization of territories purchased especially in ranching, thereby changing the use of soils and the insertion in the social scale of the regional dominant elites.

About the joint operations between the armed forces and paramilitary groups, in 2009, the declassification of secret documents disclosed by a group of researchers from George Washington University, on its Web Site called National Security Archive, was national news. With this, it can be verified that the CIA was aware that the Colombian armed forces cooperated with paramilitary groups. According to these documents, in a CIA intelligence brief released in 1994, it was determined that Colombian security forces were using "death squad tactics" in their counterinsurgency campaign. Officers had, moreover, according to that report, a history of "murder of leftist civilians in areas of guerrilla control, collaboration with paramilitaries linked to drug trafficking in attacks against suspected supporters of the guerrillas, and the murder of captured fighters" (El Espectador, 2009, para. 6).

Thus, seen from a political dimension, paramilitarism can be read as an armed reaction with a political, economic, and military alliance of local power groups in defense of the status quo; with a strong interest in preventing, through armed violence, the redefinition of the political system and social reforms, such as land distribution. A view, which is complemented by Vilma Franco's proposal (2009) of characterizing the paramilitary groups as an irregular force, which was entrusted the "counterinsurgency war and maintenance or reconfiguration of the interior order."

\section{A very brief and general timeline}




\section{Paramilitary Logics}

Opposed elites create legal forces (police, army, popol), and illegal forces (The Pájaros (The Birds), The Chulavitas, The Chusmeros (The mob), and The Bandoleros The Bandits)

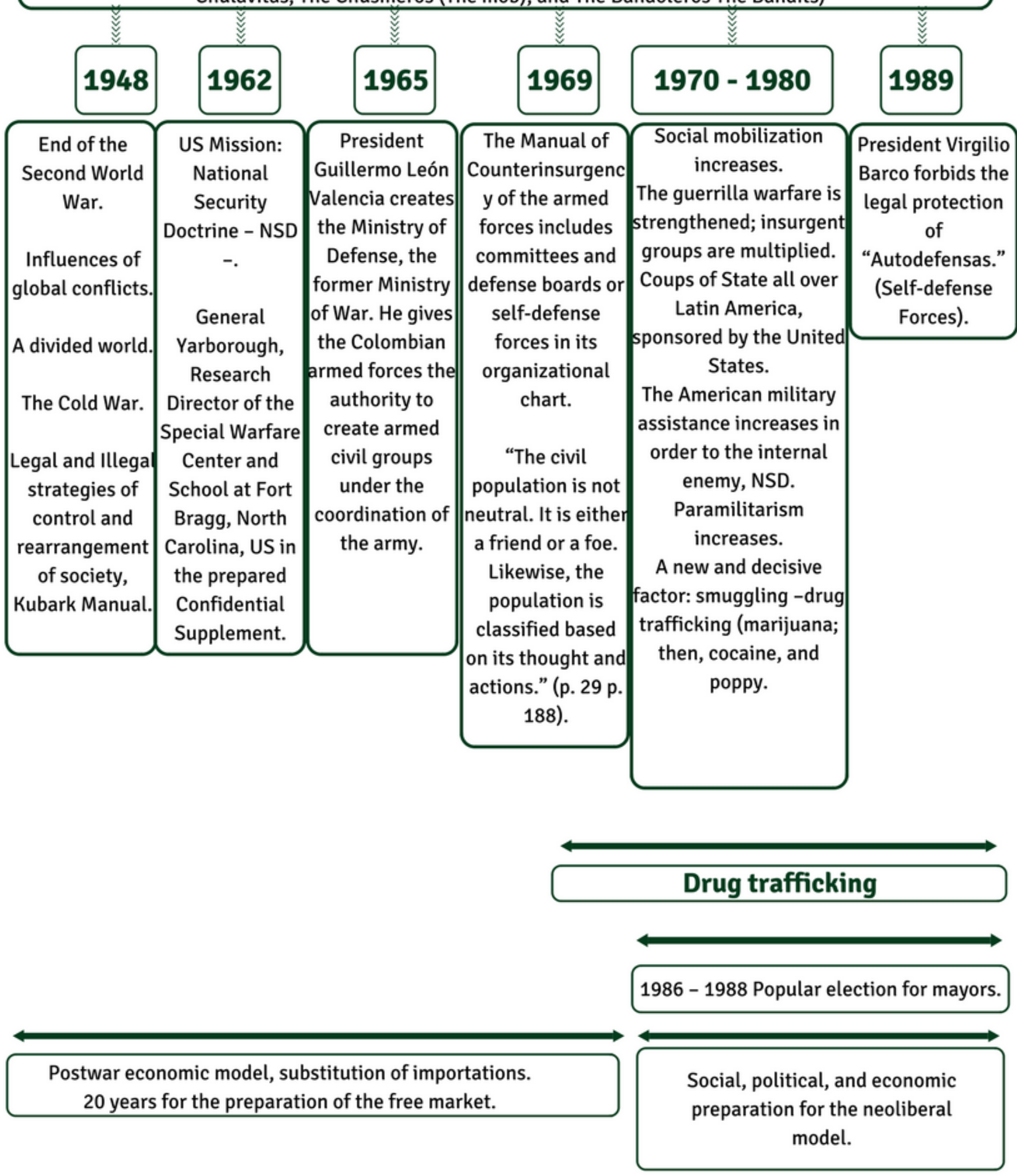

Sourse: author's elaboration 


\section{Colombian paramilitaries may differ by}

Military autonomy achieved by paramilitary groups due to their links with the drug market, as well as to the social, political, and economic support that they received from diverse regional sectors.

Even the strong alliance between paramilitary groups and the security forces, their actions went beyond counterinsurgency tasks, which were outside the scope of the army due to legal constraints related to national and international legislation of violations of human rights and the International Humanitarian Law.

They became an armed strategy armed for the accumulation of personal wealth through the dispossession of lands and their support for political and economic groups, for the accumulation through dispossession and forced displacement, as well as electoral triumphs.

The monopoly of the economy of illegal drugs, mining, smuggling, has been the loot from these groups for which it is required to maintain strong regional, political, and social alliances.

Colombia is a country with a "strong and disperse regionalization, and with an endogenous tendency, which hinders the social, economic, political integration and that of infrastructure for the formation of the national unity." (Leal, 2012, para. 2).

Functional armed and political reaction to the interests of an alliance, which brought together various sectors of the regional power, economic groups, traders and sectors of the military leadership. A framework of State legalization, which favored repression as a form of social and political conflicts, and the support of the Armed Forces in parastatal armies as a strategy to fight the internal enemy.

\section{Today: hybridization of paramilitarism as a strategy that survives}

It moves from a rural war of high intensity towards a sustained urban-rural war, for the local illegal economic control aligned with the political and economic control of each neighborhood, commune, village, township, etc.

The illegal economic activity has been hybridized with the legal one, through investments in local trade establishments, such as bakeries, gambling, "slot machines," sale and distribution of eggs, arepas (corncakes), etc.

Effective territorial control, which is indeed effective, but diluted with forced and even symbolic links, with Communal Action Boards, corporations, NGOs, etc. A struggle to capture even the contracts with the State is exercised. "By making errands", the local political participation is controlled.

This control allows alliances with local political and military authority

Circulation and legalization of money laundering is a key issue to keep in mind in this new stage. How can they circulate and legalize the money resulting from these activities?

During the devastation of the counterinsurgency war in its recent stage in parallel, a series of reforms to the State were consolidated, which made it the most faithful defender of transnational investment, gradual reforms to education, shaping it according to the "global" economic, business 
model and legal reforms occur as a result of the concentration of information in two major centers of the power media, which control what is being said, what is being thought, what is said all over the country, what is seen on T.V. and what is heard on the radio. They are a whole system of control and social direction, which capture the feeling, the thinking, the wishing, the dreaming, and the voice of a whole social mass. This is also a clear strategy of Control.

- A feeling of naturalization and learned hopelessness I created. Nothing can be done. Nothing can be changed. The best thing to do is a single struggle to achieve something, the minimum for everyone.

- A new generation grows, who seeks to disconnect their stories and, therefore, their struggles as a people.

- There follows a period of capture of territories, of large directly dedicated areas or the agro industry, or the mining-energetic activity, given to major transnational corporations.

- The actions of these groups deepens, normalizes, and naturalizes in strategic mining areas, of the agro industry, where the construction of mega-infrastructure projects is plot. They even support in transit of their productive vocation. Everything is encouraging the market to the global logic of "social and productive rearrangement of territories."

The consolidation of a prone cultural logic to the interests of the market is worrying, and that normalizing values like those of having, of accumulating, of squandering, the narco-paramilitary culture, the consolidation of policies that deindustrialized the region and that of the country, generating greater social abandonment, humanitarian crisis. It is a breeding ground in order to generate instability, which requires "another order," in the neighborhoods and communes. At the same time, the basic and higher educational system no longer form subjects, who are able to understand what is happening, to think about their history, to think other ways to build society. It only adapts the individual to the system, avoiding the critical mass, who is able to dictate terms.

Today, despite these scenarios, hope is emphasized, other ways of doing things is articulated, of building critical thinking. Various exercises for rescuing the subject are articulated. They aim at reshaping the social fabric from the very sense of communities, the defense of the public, articulating research and action. The training from other locations of what has been set up, the advocacy of common goods, of water, of the environment, of culture, of life. Networks and articulations gain a greater sense, strength, and vigor. They raise their voice gradually, from other places, different from those "known" places, which is taking place in multiple regions throughout Latin America.

\section{References}

ACA, A. C. (2003). Desplazados ¿Refugiados internos o excluidos? Medellín: ACA.

Accion Contra Minas. (2016). Definición Internacional victimas MAP MUSE. Obtenido de accioncontraminas.gov.co:

http://www.accioncontraminas.gov.co/estadisticas/Paginas/victimas-minasantipersonal.aspx 
Accion contra minas. (31 de julio de 2017). Victimas de Minas Antipersonal. Obtenido de Accion contra minas: http://www.accioncontraminas.gov.co/estadisticas/Paginas/victimas-minasantipersonal.aspx

Bastidas, W., Carrion, J., Balbin Alvarez, J. W., \& Insuasty Rodriguez, A. (2010). Las Victimas en Contextos de Violencia e Impunidad: Caso Medellín. Medellìn: Instituto Popular de Capacitaciòn.

Behar, O. (2011). El clan de Los Doce Apóstoles. Bogotá: Ícono.

Cadavid , P., Insuasty Rodriguez , A., Restrepo , L., \& Balbin , J. (2009). Victimas, violencia y despojo. medellin: IPC. Obtenido de http://web.usbmed.edu.co/usbmed/formacion/docs/victimas.pdf

CCEEU. (2016). Entre el sueño de la Pazy la continuidad de la Guerra. Informe Derechos Humanos 2016. Medellin: CCEEU.

CCOA. (20 de febrero de 2016). Generamos un ambiente propicios para los negocios. Obtenido de Camara de comercio del Oriente Antioqueño: https://www.ccoa.org.co/contenidos/categorias/oriente_antioquenio.php

CJL, C. J. (2012). Caminando en La Esperanza por Justicia y dignidad. Medellín: Nuevo Milenio.

CNMH, C. N. (2010). Silenciar la democracia. Las masacres de Remedios y Segovia 1982-1997. Bogotá: Taurus.

Corporación Jurídica Libertad. (20 de noviembre de 2012). Comisión en la Esperanza por justicia y dignidad. Medellin: Corporación Jurídica Libertad. Obtenido de Coporacion Jurídica Libertad: http://www.cjlibertad.org/files/Caminando \%20en\%201a\%20Esperanza.pdf

Gómez, J. D. (2013). Consecuencias psicosociales de la participación en escenarios de justicia transicional en un contexto de conflicto, impunidad y no-transición. El Ágora USB, 307-338.

Grisales González, D., \& Insuasty Rodriguez, A. (2016). Mineria, conflictos y derechos de las victimas. Colombia. Medellín: Editorial Kavilando.

Grisales González, D., Insuasty Rodriguez, A., \& Gutierrez Leon, E. (2013). Conflictos asociados a la gran mineria en Antioquia. El Agora USB, 13(1), 371-397. Obtenido de http://revistas.usb.edu.co/index.php/Agora/article/view/213/94

ILSA, I. L., FCSPP, F., \& MOVICE, M. (2009). Desplazamiento Forzao y retorno en San Carlos Antioquia. Una comunidad que regresa hacia el confinamiento. Bogotá: Gente Nueva.

Incoder. (s.f.). Estrategia de desarrollo rural. Obtenido de Incoder: http://www.incoder.gov.co/documentos/Estrategia $\% 20 \mathrm{de} \% 20$ Desarrollo\%20Rural/Pertile s\%20Territoriales/ADR_URABA/Documentos $\% 20 \mathrm{de} \% 20$ apoyo/DNPAtrato $\% 20 \mathrm{y} \% 20 \mathrm{Ur}$ aba/3c23PlanUraba4.pdf

Insuasty Rodriguez, A., Henao Fierro, H., Pino Franco, Y., Borja Bedoya, E., Barrera Machado, D., Sanchez Calle, D., \& Mejìa Murillo, M. (2016). Murindò. Medellìn: Kavilando.

Insuaty Rodriguez, A. (16 de marzo de 2017). "Nadie nos defendia". Exterminio de la Unión Patriótica. Colombia. Obtenido de kavilando: http://kavilando.org/index.php/2013-10-13-19-5210/confllicto-social-y-paz/5372-nadie-nos-defendia-exterminio-de-la-union-patrioticacolombia

IPC, I. P. (2015). Territorialidad, poder, conflicto y par. Medellín: IPC. 
Legiscomex.com. (14 de Agosto de 2013). ¿Aumentará la competitividad de Medellín con la construcción del Puerto de Urabá? Obtenido de legiscomex.com:

http://www.legiscomex.com/BancoMedios/Documentos $\% 20 \mathrm{PDF} /$ distribuci $\% \mathrm{C} 3 \% \mathrm{~B} 3 \mathrm{n} \% 2$ 0f $\%$ C3\%ADsica $\% 20$ internacional-puerto $\% 20 \mathrm{de} \% 20$ urab $\% \mathrm{C} 3 \% \mathrm{~A} 1$. pdf

Molano, A. (24 de Enero de 2009). La socia mandé norte. Obtenido de El Espectador: http://www.elespectador.com/choco/columna111273-socia-mande-norte

Mujica Chirinos , N., \& Rincón González , S. (2006). Concepciones del desarrollo en el Siglo XX:

Estado y Política Social. Revista de Ciencias Sociales (Ve), XII(2), 205-222. Obtenido de

http:/ / redalyc.uaemex.mx/src/inicio/ArtPdfRed.jsp?iCve=28011651002

Observatorio de Paz y Reconciliación del Oriente Antioqueño. (2006). Boletin \#1. Rionegro:

Observatorio de Paz.

Observatorio de Paz y Reconciliación del Oriente Antioqueño. (2007). Boletín \#2. Rionegro:

Observatorio de Paz.

Observatorio de Paz y Reconciliación del Oriente Antioqueño. (2008). Boletin \# 4 informe 2007.

Rionegro: Observatorio de Paz y Reconciliación del Oriente Antioqueño.

Observatorio de Paz y Reconciliación del Oriente Antioqueño. (2008). Boletín \# 6 Mapa Político en el Oriente Antioqueño 1988-2008. Rionegro: Observatorio de Paz y Reconciliación del Oriente Antioqueño.

Observatorio de Paz y Reconciliación del Oriente Antioqueño. (2008). Oriente: memoria desenterrada. Rionegro: Observatorio de Paz y Reconciliación del Oriente Antioqueño.

Ochoa, F., Smith Quintero, R., \& Villegas Botero, L. J. (2002). El sector ele $\square$ ctrico colombiano : or $\square$ genes, evolucio $\square$ ny retos o un siglo de desarrollo, 1882-1999. Bogota $\square$, D.C. : Interconexio $\square$ n Ele $\square$ ctrica S.A.

Olaya Rodríguez, C. (2012). Nunca mas contra nadie. Medellín: Cuervo Editores.

Olaya Rodriguez, C. H. (2016). Conflictos socio-ambientales en el Oriente Antioqueño. Kavilando, 8(2), 15-21. Obtenido de

http://kavilando.org/revista/index.php/kavilando/article/view/28/17

Olaya Rodriguez, C. H. (2017). El exterminio del Movimiento Cívico del Oriente de Antioquia. El Agora USB, 128-144. doi:http://dx.doi.org/10.21500/16578031.2815

Olaya, C. (2012). Nunca más contra nadie. Medellín: Cuervo Editores.

Orrego, L. P. (2008). La memoria decapitada. Espacio y estética en los asentamientos de desplazados de la ciudad de Medellin. Medellín: IDEA.

OSH, O. d. (2014). Nuestras voces sobre seguridad bumana en Medellin. Medellín: Universidad de Antioquia.

Ruíz, C. (2000). Un Pueblo que lucha: el Oriente Antioqueño.

Serrano, A. (2010). La multinacional del Crimen. La tenebrosa oficina de Envigado. Bogotá: Debate.

Suárez, J. E. (2016). Colombia nunca más Crímenes de lesa bumanidad en la comuna trece. Medellín: Léanlo.

Unidad Nacional de Atención y Reparacion a Victimas del conflicto Armado. (2016). Cirfras Unidad de Victimas. Obtenido de unidadvictimas.gov.co:

http://cifras.unidadvictimas.gov.co/Home/Vigencia 
Universidad de Antioquia \& Corporación Jurídica Libertad CJL. (2012). Crímenes de Lesa Humanidady de guerra. Medellín: Universidad de Antioquia.

Valencia Grajales, J. F., Insuasty Rodriguez, A. \&, Agudelo Arango, J. J., (2017). Elementos para una genealogía del paramilitarismo en Medellin. Medellin: Kavilando.

Valencias Grajales, J. F., Insuasty Rodriguez, A., \& Restrepo Ramirez, J. d. (2016). Elementos para una genealogía del paramilitarismo en Colombia. Medellin: Kavilando. Obtenido de http://biblioteca.clacso.edu.ar/Colombia/kavilando/20170210032121/0.pdf

Vega Cantor, R. (2012). Colombia en la geopolitica hoy. El Ágora USB, 367-402. doi:http://dx.doi.org/10.21500/16578031.81

Vega Cantor, R., \& Martín Novoa, F. (2016). Geopolítica del despojo. Bogotá: Cepa Editores.

Villa Gómez, J. D. (2013). Memoria histórica desde las Victimas del conflicto armado Construcción y reconstrucción del sujeto político. Kavilando, 5(1), 11-23. Obtenido de http://kavilando.org/revista/index.php/kavilando/article/view/96/82

Villa Gómez, J. D., \& Insuasty Rodriguez, A. (2016). Entre la participación y la resistencia: reconstrucción del tejido social desde abajo, más allá de la lógica de reparación estatal. El Agora USB, 453-478.

Villa Gómez, J. D., \& Insuasty Rodriguez, A. (2016). Significados en torno a la indemnización y la restitución en víctimas del conflicto armado en el municipio de San Carlos. El Ágora USB, 16(1), 165-191. Obtenido de http:/ / revistas.usb.edu.co/index.php/Agora/article/view/2171/1899

Viva la Ciudadanía. (20 de enero de 2007). La ruta de la expansión paramilitar y la transformacion de Antioquia. Obtenido de Viva la ciudadania: http://viva.org.co/cajavirtual/svc0080/articulo10.pdf 